\title{
Fabrication and characterization of ultra-high resolution multilayer-coated blazed gratings
}

\author{
Dmitriy L. Voronov, * Erik H. Anderson, Rossana Cambie, Scott Dhuey, Eric M. Gullikson, Farhad Salmassi, \\ Tony Warwick, Valeriy V. Yashchuk, and Howard A. Padmore \\ Lawrence Berkeley National Laboratory, Berkeley, CA 94720 \\ *E-mail: dlvoronov@lbl.gov
}

\begin{abstract}
Multilayer coated blazed gratings with high groove density are the most promising candidate for ultra-high resolution soft $\mathrm{x}$-ray spectroscopy. They combine the ability of blazed gratings to concentrate almost all diffraction energy in a desired high diffraction order with high reflectance soft x-ray multilayers. However in order to realize this potential, the grating fabrication process should provide a near perfect groove profile with an extremely smooth surface of the blazed facets. Here we report on successful fabrication and testing of ultra-dense saw-tooth substrates with 5,000 and 10,000 lines $/ \mathrm{mm}$.
\end{abstract}

Key words: diffraction grating, diffraction efficiency, blazed grating, multilayer coating, e-beam lithography, wet anisotropic etch, EUV, soft x-rays, atomic force microscopy.

\section{Introduction}

Soft x-ray spectroscopy techniques like Resonance Inelastic X-ray Scattering require a very high spectral resolution of $10^{4}-10^{6}$ [1]. This imposes very high requirements for the diffraction gratings which are a key component of x-ray spectrometers. Traditionally grazing incidence gratings are used operating in a $1^{\text {st }}$ diffraction order which has some limitation in terms of spectral resolution [2] due to slope error, size of the spectrograph and throughput, all of which are eliminated by high-order high-density gratings. The development of these ideas closely follows those adopted for highresolution spectroscopy in the optical regime.

These obstacles can be overcome with multilayer-coated blazed gratings (MLG), which according to simulations can deliver very high efficiency in a high diffraction order [3]. The high dispersion mitigates problems due to slope error, reduces geometric aberrations to the larger scattering angle, increases throughput and results in a compact design [4]. Recently we reported the successful fabrication of the 5,000 lines/mm MLG for EUV [5], which is a prototype of high-resolution soft xray gratings. The saw-tooth substrate with the period of $200 \mathrm{~nm}$ was fabricated with a microfabrication process based on wet anisotropic etch of silicon single crystals, and then coated with a $\mathrm{Mo} / \mathrm{Si}$ multilayer. In this work we scale the process towards a shorter period of $100 \mathrm{~nm}$ to obtain an MLG with a groove density of 10,000 lines/mm. We characterize the gratings and compare their quality to reveal the impact of the period scaling on performance. We also investigate the impact of different multilayers on grating parameters.

\section{Experiment}

Two saw-tooth substrates with the blaze angle of 6 degrees were fabricated form identical silicon wafers with a micro-fabrication process based on wet anisotropic etch of silicon [6]. The surface of the wafers had a 6 degree inclination from (111) planes of the silicon crystal lattice. High-resolution lithography techniques were used to create periodical pattern on the surface of the wafers. Fabrication of the grating \#1 with the period of $200 \mathrm{~nm}$ used scanning beam interference lithography 
[7], and the $100 \mathrm{~nm}$ period pattern for the grating \#2 was produced with e-beam lithography. The micro-fabrication process described earlier [8] was applied to the wafers to form a saw-tooth profile of the substrates.

The saw-tooth substrates were coated with multilayers using dc-magnetron sputtering. In order to provide the blazed conditions for a particular diffraction order, the d-spacing of the multilayers was chosen according to the ratio $d \sin \phi \Delta=\mathrm{m}$, where $d$ is a grating period, $\phi$ is a blaze angle, $\Delta$ is a $\mathrm{d}$ spacing of the multilayer, and $m$ is a number of a blazed diffraction order of the grating. The grating \#1 was covered by a Mo/Si ML with $\Delta=7 \mathrm{~nm}$ which is about 3 times smaller than the grating groove depth. In this way the grating \#1 is optimized for the $3^{\text {rd }}$ diffraction order under the blazed conditions. The grating \#2 was deposited with a Al/Zr ML with $\Delta=10.25 \mathrm{~nm}$, which equals to the grating groove depth, to provide blazing for the $1^{\text {st }}$ diffraction order. The $\mathrm{Mo} / \mathrm{Si}$ and $\mathrm{Al} / \mathrm{Zr}$ multilayers consisted of 30 and 20 bi-layers respectively, so the total thickness of the MLs was approximately $200 \mathrm{~nm}$. The witness $\mathrm{Mo} / \mathrm{Si}$ and $\mathrm{Al} / \mathrm{Zr}$ multilayers were deposited on flat silicon substrates simultaneously with the corresponding gratings.

\section{Results and discussion}

The micro-fabrication process results in triangular groove profiles for both the gratings. Threedimensional AFM images of the saw-tooth substrates \#1 and \#2 are shown in Fig. 1a and 1b respectively. Both gratings have similar groove profiles shaped with 6-degree tilted blaze facet and short highly tilted anti-blazed facet. AFM measurements confirmed that surface roughness measured over a $1 \mu \mathrm{m}^{2}$ area does not exceed $0.3 \mathrm{~nm}$ rms for both the gratings. These data show that the microfabrication process was successfully scaled down to $100 \mathrm{~nm}$ period.

After the deposition of the Mo/Si multilayer the groove profile of the grating \#1 (200 nm period) underwent substantial changes (Fig. 2a). The grating grooves became smoothed and rounded especially in the vicinity of anti-blazed facets. Nevertheless the surface of the blazed facets remains stable and relatively flat and maintains the 6-degree slope.

Much more dramatic changes of the groove profile are observed for the grating \#2 (100 nm period) after the Al/Zr ML deposition (Fig. 2b). The grating surface became substantially smoothed, initially triangular grooves turned to sine-like ones, and the groove depth reduced significantly. Initially high asymmetry in slope and dimensions the blazed and anti-blazed facets is wiped out, making them almost indistinguishable.

The profile change is a result of a complicated interplay of smoothing and shadowing processes taking place during the ML deposition. Evolution of the Mo/Si stack structure during the ML growth onto the $200 \mathrm{~nm}$ saw-tooth substrate were discussed earlier [9].

The AFM data on groove profile (Fig. 1-2) can be used for characterization of smoothing properties of the multilayers. Although the gratings \#1 and \#2 have different periods, the smoothing ability of the $\mathrm{Mo} / \mathrm{Si}$ and $\mathrm{Al} / \mathrm{Zr}$ multilayers can be compared in a Power Spectral Density (PSD) domain where the difference in grating periods is not relevant. The PSD spectra for the grating \#1 and \#2 before and after ML deposition are shown in Fig. 3 and Fig. 4 respectively. The spectra have sharp peaks corresponding to Fourier harmonics of the groove profiles; the frequency of the peaks is multiple to the groove density of the gratings. Deposition of the multilayers results in suppression of highfrequency harmonics, which is much more pronounced for $\mathrm{Al} / \mathrm{Zr}$ multilayer. Intensity of the 
$0.01 \mathrm{~nm}^{-1}$ harmonic corresponding to $100 \mathrm{~nm}$ spatial wavelength reduces by a factor of 2 for $\mathrm{Mo} / \mathrm{Si}$ and by a factor of 5 for $\mathrm{Al} / \mathrm{Zr}$ multilayers. High frequency harmonics still survive after the $\mathrm{Mo} / \mathrm{Si}$ deposition, while $\mathrm{Al} / \mathrm{Zr}$ multilayer suppress completely all spatial frequencies higher than $0.02 \mathrm{~nm}^{-1}$. This difference indicates the much higher mobility of surface atoms during $\mathrm{Al} / \mathrm{Zr}$ deposition compared to $\mathrm{Mo} / \mathrm{Si}$ multilayer.

The smoothing ability is a well known property of the multilayers deposited on flat substrates [10, 11]. It is caused by surface diffusion which is a dominant relaxation process for the case of magnetron sputtering. The smoothing provides suppression of the surface roughness of flat substrates for high spatial frequencies, $\mathrm{f}>0.02 \mathrm{~nm}^{-1}$ [11]. However, impact of the smoothing extends to much lower frequencies of 0.01 and $0.005 \mathrm{~nm}^{-1}$ for the saw-tooth substrates (Fig. 3 and Fig. 4). This can be explained by impact of substrate relief on surface atom mobility.

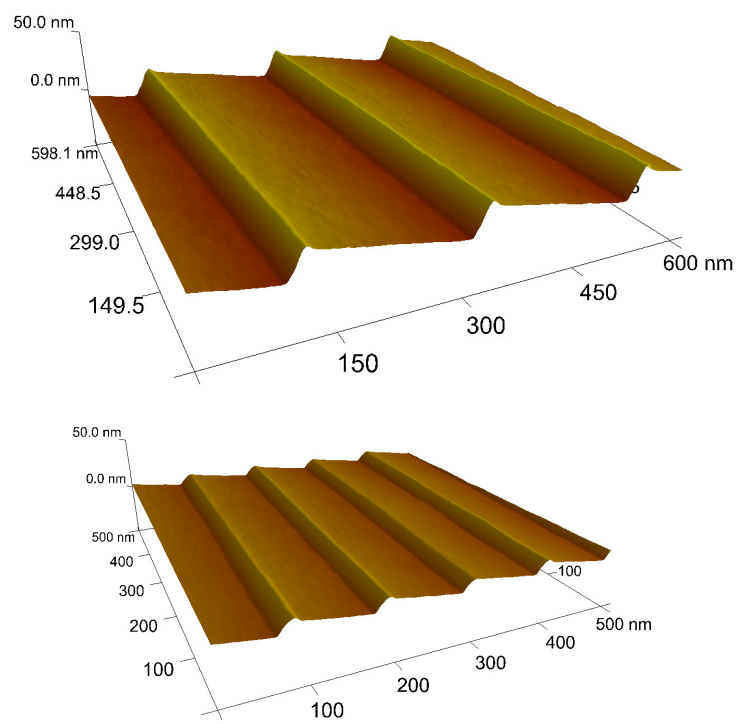

Fig. 1. AFM images of the saw-tooth silicon substrates \#1 (a) and \#2 (b) with groove density of 5,000 and 10,000 lines/mm respectively 

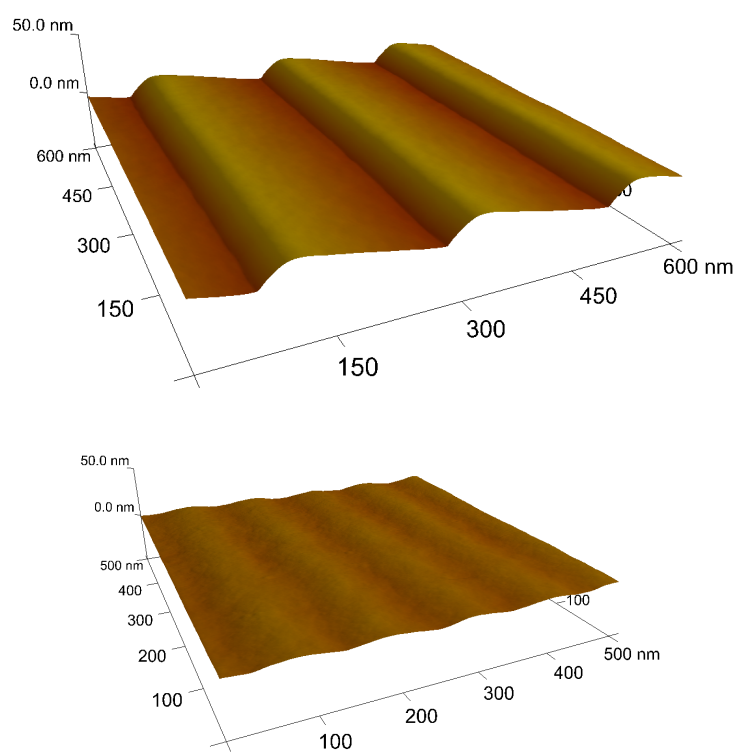

Fig. 2. AFM images of the blazed gratings \#1 (a) and \#2 (b) after deposition of Mo/Si and $\mathrm{Al} / \mathrm{Zr}$ multilayers respectively

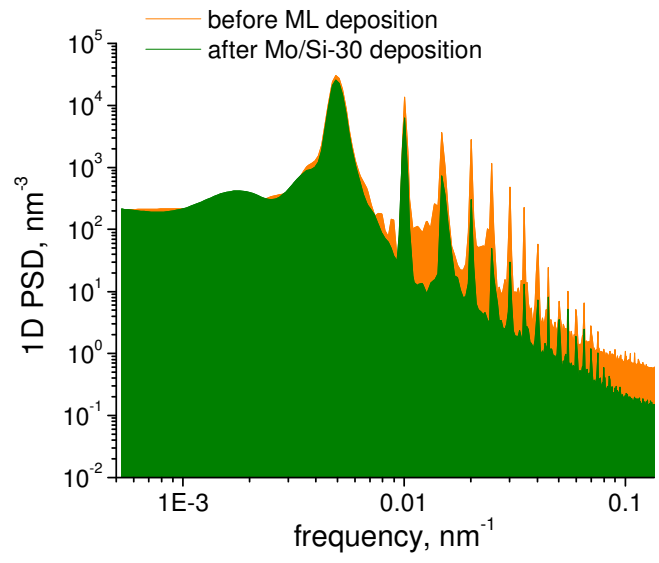

Fig. 3. One dimensional PSD spectra of the gratings \#1 before and after deposition of the $\mathrm{Mo} / \mathrm{Si}$ multilayer. 


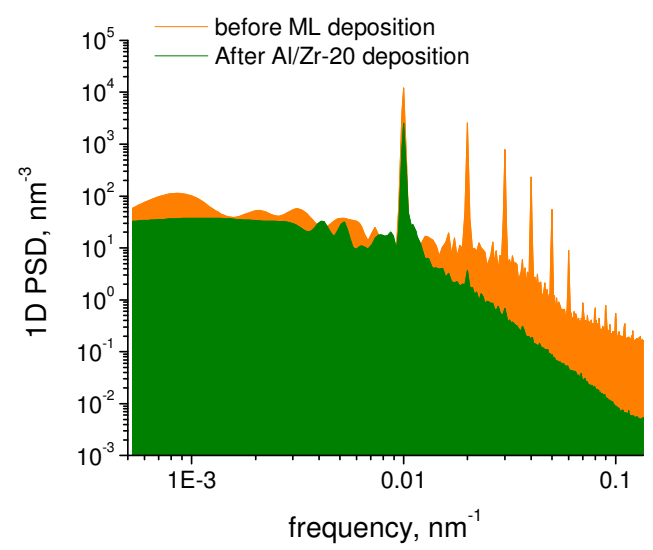

Fig. 4. One dimensional PSD spectra of the gratings \#2 before and after deposition of the $\mathrm{Al} / \mathrm{Zr}$ multilayer.

The smoothing of the groove profile results in deterioration of the blazing performance and a reduction of diffraction efficiency. A number of diffraction orders, which should be suppressed for an ideal blazed grating, appeared on the diffraction pattern of the grating \#1 (Fig. 5). At the same time the efficiency of the $3^{\text {rd }}$ blazed order reduces compared to the witness Mo/Si reflectance of $64 \%$ by a factor of 0.6 , but it is still rather high because a large portion of the blazed facets survived after deposition.

The grating \#2 that suffered much more from the smoothing demonstrates efficiency of the $1^{\text {st }}$ blaze order of $13 \%$, which is smaller than witness $\mathrm{Al} / \mathrm{Zr}$ reflectance of $48 \%$ by a factor of 4 . This is due to the large variation of the blaze angle across the grating groove so that the diffracted energy is directed to the zero and negative $1^{\text {st }}$ orders, which have efficiency comparable to the one for the blazed $1^{\text {st }}$ order. 


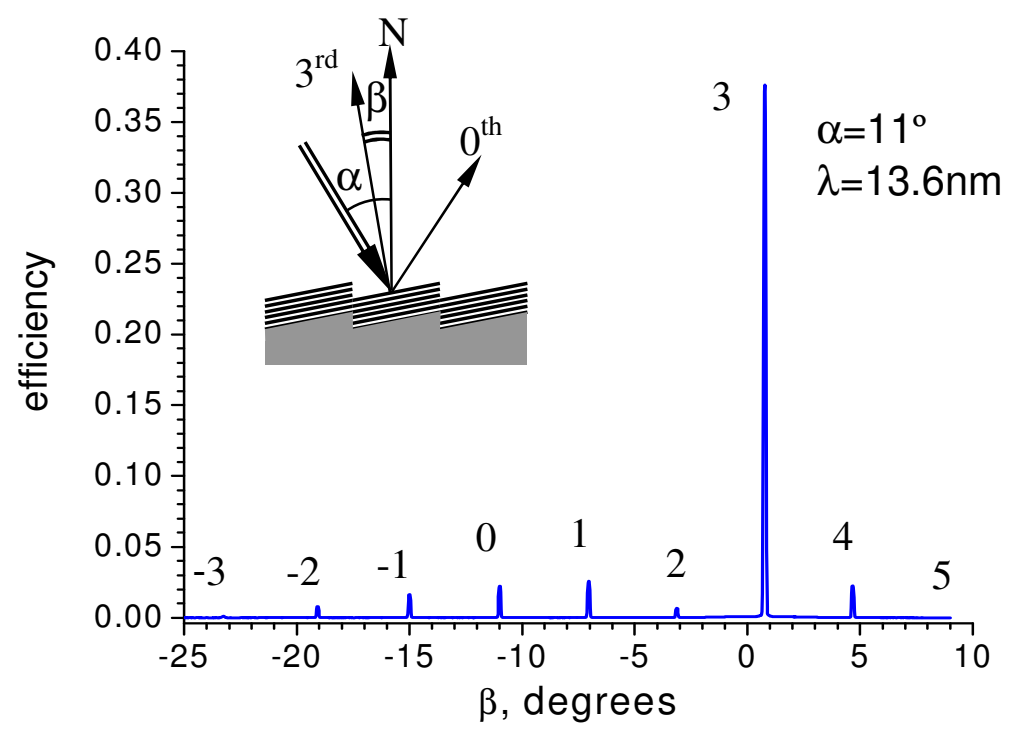

Fig. 5. Diffraction from grating \#1 coated with a Mo/Si multilayer at the incident angle of $11^{\circ}$, and the wavelength of $13.6 \mathrm{~nm}$.

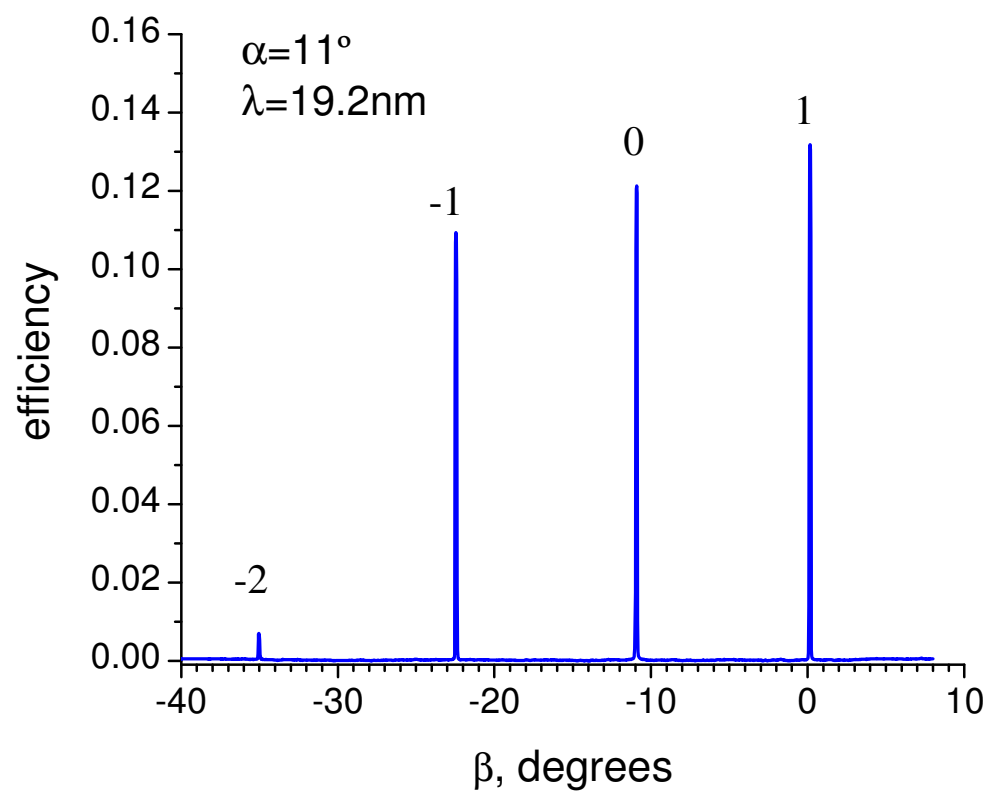

Fig. 6. Diffraction from grating \#2 coated with an $\mathrm{Al} / \mathrm{Zr}$ multilayer at the incident angle of $11^{\circ}$, and the wavelength of $19.2 \mathrm{~nm}$. 


\section{Summary}

The fabrication process for high quality saw-tooth substrates was successfully scaled towards ultrashort grating period of $100 \mathrm{~nm}$. The gratings have near-perfect triangular groove profile and very smooth surface of blaze facets.

However the deposition of a multilayer on the saw-tooth substrates results in significant smoothing of the groove profile, which results in reduction of the MLG performance. It was found that $\mathrm{Al} / \mathrm{Zr}$ multilayers have much more pronounced smoothing ability compared to Mo/Si ML. The process of the deposition of multilayers on the highly corrugated surfaces of the saw-tooth substrates imposes new conditions on deposition and elemental selection. Clearly some form of collimated deposition will reduce the transverse energy of atoms to a level much lower than in magnetron sputtering. However some degree of transverse energy is required for smoothing on the plane facet surfaces, so reduction of this energy to zero, if possible, would result in unacceptably rough surfaces. In addition, there is a finite limit to the transverse energy, given by the interaction of the depositing atom and the atomic structure of the surface. Achieving a balance between local roughness and local shape error will require a more detailed understanding of surface mobility on systems of relevance to multilayer reflectors.

This work was supported by the U. S. Department of Energy under contract number DE-AC02$05 \mathrm{CH} 11231$.

\section{Disclaimer}

This document was prepared as an account of work sponsored by the United States Government. While this document is believed to contain correct information, neither the United States Government nor any agency thereof, nor The Regents of the University of California, nor any of their employees, makes any warranty, express or implied, or assumes any legal responsibility for the accuracy, completeness, or usefulness of any information, apparatus, product, or process disclosed, or represents that its use would not infringe privately owned rights. Reference herein to any specific commercial product, process, or service by its trade name, trademark, manufacturer, or otherwise, does not necessarily constitute or imply its endorsement, recommendation, or favoring by the United States Government or any agency thereof, or The Regents of the University of California. The views and opinions of authors expressed herein do not necessarily state or reflect those of the United States Government or any agency thereof or The Regents of the University of California.

\section{References}

[1] Workshop on "Soft X-Ray Science in the Next Millennium: The Future of PhotonIn/Photon-Out Experiments." (Pikeville, Tennessee March 15-18, 2000), http://www.phys.utk.edu/WPWebSite/ewp_workshop_X-Ray_Report.pdf

[2] G. Ghiringhelli, A. Piazzalunga, C. Dallera, G. Trezzi, L. Braicovich, T. Schmitt, V.N. Strocov, R. Betemps, L. Patthey, X. Wang, M. Grioni, Rev. Sci. Instrum. 77 (2006) 113108

[3] D.L. Voronov, R. Cambie, R.M. Feshchenko, E.M. Gullikson, H.A. Padmore, A.V. Vinogradov, V.V. Yashchuk, Proc. SPIE 6705 (2007) 67050E 
[4] T. Warwick, H. Padmore, D. Voronov, V. Yashchuk, CP1234, The 10th International Conference on Synchrotron Radiation Instrumentation, R.Carrett, I. Gentle, K. Nugent, and S. Wilkins, AIP (2010) 776

[5] D.L. Voronov, M. Ahn, E.H. Anderson, R. Cambie, Ch.-H. Chang, E.M. Gullikson, R.K. Heilmann, F. Salmassi, M.L. Schattenburg, T. Warwick, V.V. Yashchuk, L. Zipp, H.A. Padmore, Opt. Lett. 35 (2010) 2615-2618

[6] P. Philippe, S. Valette, O. Mata Mendez, D. Maystre, Appl. Opt. 24 (1985) 1006-1011

[7] R.K. Heilmann, C.G. Chen, P.T. Konkola, M.L. Schattenburg, Nanotechnology 15 (2004) S504-S511

[8] D.L.Voronov, E.H. Anderson, R. Cambie, F. Salmassi, E.M. Gullikson, V.V. Yashchuk, H.A. Padmore, M. Ahn, Ch.-H. Chang, R.K. Heilmann, M.L. Schattenburg, Proc. SPIE 7448 (2009) 74480J

[9] D.L.Voronov, E.H. Anderson, R. Cambie, F. Salmassi, E.M. Gullikson, V.V. Yashchuk, H.A. Padmore, M. Ahn, Ch.-H. Chang, R.K. Heilmann, M.L. Schattenburg, Proc. SPIE 7802 (2010) 780207

[10] D.G. Stearns, D.P. Gaines, D.W. Sweeney, E.M. Gullikson, J. Appl. Phys., 84 (1998)10031028

[11] E. Spiller, S. Baker, E. Parra, C. Tarrio, Proc. SPIE 3767 (1999) 143-153 